\title{
Picard Groups on Moduli of K3 Surfaces with Mukai Models
}

\author{
Francois Greer ${ }^{1}$, Zhiyuan $\mathbf{L i}^{1}$, and Zhiyu Tian ${ }^{2}$ \\ ${ }^{1}$ Department of Mathematics, Building 380, Stanford, CA 94305, USA and \\ ${ }^{2}$ Department of Mathematics, California Institute of Technology, \\ Pasadena, CA 91125, USA
}

Correspondence to be sent to: zli2@stanford.edu

We discuss the Picard group of the moduli space $\mathcal{K}_{g}$ of quasi-polarized K3 surfaces of genus $g \leq 12$ and $g \neq 11$. In this range, $\mathcal{K}_{g}$ is unirational, and a general element in $\mathcal{K}_{g}$ is a complete intersection with respect to a vector bundle on a homogenous space, by the work of Mukai. In this paper, we find generators for the Picard group $\operatorname{Pic}_{\mathbb{Q}}\left(\mathcal{K}_{g}\right)$ using the Noether-Lefschetz (NL) theory. This verifies the NL conjecture on the moduli of K3 surfaces in these cases.

\section{Introduction}

It is well known that the moduli space $\mathcal{M}_{g}$ of smooth projective curves of genus $g$ is a quasi-projective variety with Picard number one for $g \geq 3$ (cf. [9]). The Picard group $\operatorname{Pic}_{\mathbb{Q}}\left(\mathcal{M}_{g}\right)$ with rational coefficients is generated by the first Chern class of the Hodge bundle. In the moduli theory of higher-dimensional varieties, the primitively polarized K3 surface of genus $g$ can be viewed as a 2-dimensional analog of genus $g$ smooth projective curve, and it is natural for us to study the Picard group of its moduli space $\mathcal{K}_{g}^{\circ}$.

Unlike in the curve case, the rank of $\operatorname{Pic}\left(\mathcal{K}_{g}^{\circ}\right)$ is no longer constant; it has been shown by $\mathrm{O}^{\prime} \mathrm{Grady}[21]$ that $\operatorname{rank}\left(\operatorname{Pic}\left(\mathcal{K}_{g}^{\circ}\right)\right)$ increases to infinity as $g$ is increasing (see also [16, Section 7]). Besides the Hodge line bundle, there are many other natural 
divisors on $\mathcal{K}_{g}^{\circ}$ coming from geometry. Actually, the Noether-Lefschetz (NL) locus of $\mathcal{K}_{g}^{\circ}$ parameterizing $\mathrm{K} 3$ surfaces with Picard number $>1$ is a union of countably many irreducible divisors by the Hodge theory. We call them the $N L$ divisors on $\mathcal{K}_{g}^{\circ}$. In this paper, we study the Picard group on $\mathcal{K}_{g}^{\circ}$ for $g \leq 12(g \neq 11)$ and find its generators in terms of NL-divisors on $\mathcal{K}_{g}^{\circ}$.

For convenience, instead of working on $\mathcal{K}_{g}^{\circ}$, we will study the moduli space $\mathcal{K}_{g}$ of primitively quasi-polarized K3 surfaces of genus $g$, which is more natural from a Hodge theoretic point of view. It is known that $\mathcal{K}_{g}$ is a locally Hermitian symmetric variety, by the Torelli theorem, and the complement $\mathcal{K}_{g} \backslash \mathcal{K}_{g}^{\circ}$ is a divisor parameterizing $K 3$ surfaces containing a (-2)-exceptional curve. In this setting, we define the NL divisors on $\mathcal{K}_{g}$ as follows: given $d, n \in \mathbb{Z}$, let $D_{d, n}^{g} \subset \mathcal{K}_{g}$ be the locus of those K3 surfaces $S \in \mathcal{K}_{g}$ whose Picard lattice $\operatorname{Pic}(S)$ contains a primitive rank 2 sublattice

\begin{tabular}{c|c|c} 
& $L$ & $\beta$ \\
\hline$L$ & $2 g-2$ & $d$ \\
\hline$\beta$ & $d$ & $n$
\end{tabular}

where $L$ is the primitive quasi-polarization of $S$ and $\beta \in \operatorname{Pic}(S)$. Each NL divisor $D_{d, n}^{g}$ is irreducible by [22]. In [16], Maulik and Pandharipande conjectured that the divisors $\left\{D_{d, n}^{g}\right\}$ span the group $\operatorname{Pic}_{\mathbb{Q}}\left(\mathcal{K}_{g}\right)$ with rational coefficients. Our main result is the following theorem.

Theorem 1.1. The Picard group of $\mathcal{K}_{g}$ with rational coefficients is spanned by NL divisors for $g \leq 10$ and $g=12$. Moreover, the basis of $\operatorname{Pic}_{\mathbb{Q}}\left(\mathcal{K}_{g}\right)$ of $6 \leq g \leq 10$, is given by:

- $g=6,\left\{D_{0,-2}^{6}, D_{5,2}^{6}, D_{k, 0}^{6}, k=1, \ldots 4\right\}$.

- $g=7,\left\{D_{0,-2}^{7}, D_{5,2}^{7}, D_{6,2}^{7}, D_{k, 0}^{7}, k=1, \ldots 4\right\}$.

- $g=8,\left\{D_{0,-2}^{8}, D_{6,2}^{8}, D_{7,2}^{8} D_{k, 0}^{8}, k=1, \ldots 4\right\}$.

- $g=9,\left\{D_{0,-2}^{9}, D_{6,2}^{9}, D_{7,2}^{9} D_{k, 0}^{9}, k=1, \ldots 5\right\}$.

- $g=10,\left\{D_{0,-2}^{10}, D_{7,2}^{10}, D_{8,2}^{10}, D_{9,4}^{10}, D_{k, 0}^{10}, k=1, \ldots 5\right\}$.

For $g=12$, the group $\operatorname{Pic}_{\mathbb{Q}}\left(\mathcal{K}_{12}\right)$ is generated by

$$
\left\{D_{0,-2}^{12}, D_{7,2}^{12}, D_{8,2}^{12}, D_{9,2}^{12}, D_{10,4}^{12}, D_{11,4}^{12}, D_{k, 0}^{12}, k=1, \ldots 6\right\} .
$$

Remark 1.2. The rank of $\operatorname{Pic}\left(\mathcal{K}_{12}\right)$ is 11 , so there is a linear relation between the generators in (1.2). See Remark 4.2 for more details. 
When $2 \leq g \leq 5$, a general K3 surface in $\mathcal{K}_{g}$ is a double cover of $\mathbb{P}^{2}(g=2)$ or a complete intersection in $\mathbb{P}^{g}$, and the assertion can be found in [15, 22, 25, 26]. In these cases, the proof relies on the explicit construction of the moduli space of the corresponding complete intersections via geometric invariant theory (GIT). If $g$ is $>5$, the general K3 surface of genus $g$ is no longer a complete intersection in $\mathbb{P}^{g}$, but it can be interpreted as a complete intersection with respect to certain vector bundles on homogenous spaces, for $g$ in the range of our theorem, so there ought to be a similar construction.

\section{Geometry of K3 Surfaces}

In this section, we review Mukai's work on the projective models of general low genus quasi-polarized K3 surfaces. We give a precise characterization, in terms of the Picard lattice, of the (nongeneral) K3 surfaces lying outside the locus of Mukai models. We also include a few examples to illustrate the phenomenon. Throughout this paper, we work over complex numbers.

\subsection{Notations}

Let $(S, L)$ be a primitively quasi-polarized K3 surface of genus $g$, that is, $L \in \operatorname{Pic}(S)$ is big and nef with $L^{2}=2 g-2$. The middle cohomology $H^{2}(S, \mathbb{Z})$ is an even unimodular lattice of signature $(3,19)$ under the intersection form $\langle$,$\rangle and is isometric to$

$$
U^{\oplus 3} \oplus\left(-E_{8}\right)^{\oplus 2},
$$

by the classification of even unimodular indefinite lattices, where $U$ is the hyperbolic lattice of rank 2 and $E_{8}$ is the positive-definite lattice associated to the Lie group of the same name. Let $\Lambda_{g}:=C_{1}(L)^{\perp}$ be the orthogonal complement, which is an even lattice of signature $(2,19)$. The isometry class of $\Lambda_{g}$ is independent of $(S, L)$ because the group of isometries of $U^{\oplus 3} \oplus\left(-E_{8}\right)^{\oplus 2}$ acts transitively on the set of primitive vectors of square $(2 g-2)$ (cf. [7, Proposition 3.3]).

The period domain $\mathcal{D}_{g}$ associated to $\Lambda_{g}$ can be realized as a connected component of

$$
\mathcal{D}_{g}^{ \pm}:=\left\{v \in \mathbb{P}\left(\Lambda_{g} \otimes \mathbb{C}\right) \mid\langle v, v\rangle=0,\langle v, \bar{v}\rangle>0\right\}
$$

The monodromy group

$$
\Gamma_{g}=\left\{g \in \operatorname{Aut}\left(\Lambda_{g}\right)^{+} \mid g \text { acts trivially on } \Lambda_{g}^{\vee} / \Lambda_{g}\right\},
$$


naturally acts on $\mathcal{D}_{g}$, where $\operatorname{Aut}\left(\Lambda_{g}\right)^{+}$denotes the connected component of the identity of $\operatorname{Aut}\left(\Lambda_{g}\right)$. According to the Global Torelli theorem for K3 surfaces, there is an isomorphism

$$
\mathcal{K}_{g} \cong \mathcal{D}_{g} / \Gamma_{g}
$$

via the period map. Hence, $\mathcal{K}_{g}$ is a locally Hermitian symmetric variety with only finite quotient singularities, and is thus $\mathbb{Q}$-factorial. Each NL divisor $D_{d, n}^{g}$ can be considered as the quotient of a codimension 1 subdomain in $\mathcal{D}_{g}$.

As we will use later, we also define the divisors on $\mathcal{K}_{g}$ by specifying a curve class (cf. [16]): given the data $d, n$, let $\mathcal{C}_{d, n}^{g}$ be the locus parameterizing the K3 surfaces containing a class $\beta$ with $\beta^{2}=n$ and $L \cdot \beta=d$. The divisor $\mathcal{C}_{d, n}^{g}$ is supported on a collection of NL divisors $D_{d^{\prime}, n^{\prime}}^{g}$ satisfying $\Delta_{d^{\prime}, n^{\prime}}^{g}=k^{2} \Delta_{d, n}^{g}$ for some $k \in \mathbb{Z}$, where $\Delta_{d, n}^{g}$ is the determinant of the lattice $\Lambda_{d, n}^{g}$. It is not hard to see that the span of two sets $\left\{D_{d, n}^{g}\right\}$ and $\left\{\mathcal{C}_{d, n}^{g}\right\}$ are the same, and $\mathcal{K}_{g} \backslash \mathcal{K}_{g}^{\circ}=\mathcal{C}_{0,-2}^{g}$.

\subsection{Mukai models}

Let $(S, L)$ be a smooth quasi-polarized K3 surface of genus $g$. The linear system $|L|$ defines a map $\psi_{L}$ from $S$ to $\mathbb{P}^{g}$. The image of $\psi_{L}$ is called a projective model of $S$. If $\psi_{L}$ is birational to its image, then $\psi_{L}(S)$ is a degree $2 g-2$ surface in $\mathbb{P}^{g}$ with at worst rational double points.

Remark 2.1. Suppose that, $\psi_{L}$ is birational. If the K3 surface $S$ contains a $(-2)$ exceptional curve, the morphism $\psi_{L}$ contracts this exceptional curve and the image $\psi_{L}(S)$ has a rational double point. Thus, it is easy to see that $\psi_{L}(S)$ is singular only if $(S, L) \in \mathcal{K}_{g} \backslash \mathcal{K}_{g}^{\circ}$.

As we mentioned in the introduction, when $6 \leq g \leq 12(g \neq 11)$, general members in $\mathcal{K}_{g}$ are no longer complete inspections in $\mathbb{P}^{g}$, but can be interpreted as complete intersections with respect to vector bundles on homogenous spaces in the following sense. Let $E$ be a rank $r$ vector bundle on a smooth projective variety $X$ with local frame $\left\{e_{1}, \ldots, e_{r}\right\}$. A global section

$$
s=\sum_{i=1}^{r} f_{i} e_{i} \in H^{0}(X, E), \quad f_{i} \in \mathcal{O}_{X},
$$


is nondegenerate at $x$ if $s(x)=0$ and $\left(f_{1}, \ldots, f_{r}\right)$ is a regular sequence. The section $s$ is nondegenerate if it is nondegenerate at every point of $(s)_{0}$, where $(s)_{0}$ the zero locus of the section $s$.

A subscheme $Y$ is a complete intersection with respect to $E$ if $Y$ is the zero locus of a nondegenerate global section of $W$. In particular, one can view the complete intersection of $r$-hypersurfaces $H_{1}, H_{2}, \ldots, H_{r}$ in $X$ as the complete intersection with respect to the vector bundle

$$
E=\mathcal{O}_{X}\left(H_{1}\right) \oplus \mathcal{O}_{X}\left(H_{2}\right) \cdots \oplus \mathcal{O}_{X}\left(H_{r}\right)
$$

on $X$.

Now we review Mukai's Brill-Noether (BN) theory on K3 surfaces, which essentially gives us the classification of the projective models of general K3 surfaces. For simplicity of the discussion, we may only consider the polarized K3 surfaces in $\mathcal{K}_{g}^{\circ}$.

Definition 2.2. A polarized K3 surface $(S, L)$ of genus $g$ is BN general if the inequality $h^{0}(M) h^{0}(N)<h^{0}(L)$ holds for any pair $(M, N)$ of nontrivial line bundles such that $M \otimes N \cong L$.

Remark 2.3. The BN theory on $\mathrm{K} 3$ surfaces is analogous to the BN theory on curves. Actually, if a smooth curve $C \in|L|$ is BN general, then the polarized K3 surface $(S, L)$ is BN general (cf. [8]).

When $(S, L)$ is BN general of genus $g$, for any two integers $r, s$ with $r s=g$, Mukai [19] shows that there exists a rigid and stable vector bundle $V_{r}$ on $S$ of rank $r$ such that $V_{r}$ is globally generated and $\wedge^{r} V_{r} \cong L$. The higher cohomology of $V_{r}$ vanishes and $\operatorname{dim} H^{0}\left(S, V_{r}\right)=r+s$. Then there is a map

$$
\Phi_{V_{r}}: S \rightarrow \operatorname{Gr}\left(r, H^{0}\left(S, V_{r}\right)^{\vee}\right)
$$

Remark 2.4. For $g=6,8,9,10,12$, the values of $r, s$ chosen by Mukai are

\begin{tabular}{cccccc}
\hline$g$ & 6 & 8 & 9 & 10 & 12 \\
\hline$r s$ & $2 \cdot 3$ & $2 \cdot 4$ & $3 \cdot 3$ & $2 \cdot 5$ & $3 \cdot 4$ \\
\hline
\end{tabular}

In the case $g=7$, Mukai chose the rank 5 vector bundle $V_{7}$ with $\wedge{ }^{5} E_{7}=L^{\otimes 2}$. The linear system $\left(V_{7}, H^{0}\left(S, V_{7}\right)\right)$ embeds the K3 surface $S$ into a Grassmannian $\operatorname{Gr}(5,10)$, whose image is contained in the isotropic Grassmannian $\operatorname{IGr}(5,10)$. 
For $6 \leq g \leq 12$ and $g \neq 11$, Mukai has shown that the image of $\Phi_{E_{r}}$ can be described as complete intersections with respect to a vector bundle on some homogenous subspace in $\operatorname{Gr}\left(r, H^{0}\left(S, V_{r}\right)\right)^{\vee}$. Here we just summarize Mukai's results in [19].

Theorem 2.5. If a primitively polarized K3 surface $(S, L)$ of genus $6 \leq g \leq 10$ or $g=12$ is $\mathrm{BN}$ general, then it is isomorphic to a complete intersection with respect to a vector bundle $E_{g}$ in a homogenous space $X_{g}$ via $\psi_{L}$, where $X_{g}$ and the images $\psi_{L}(S)$ are listed:

- $g=6: X_{6}=\operatorname{Gr}(2,5)$ and $E_{6}=\mathcal{O}_{X_{6}}(1)^{\oplus 3} \oplus \mathcal{O}_{X_{6}}(2), \psi_{L}(S)$ is a complete intersection of a quadric and a codimension 3 linear section in $X_{6}$;

- $g=7: X_{7}$ is the isotropic Grassmannian $\operatorname{IGr}(5,10)$ and $E_{7}=\mathcal{O}_{X_{7}}(1)^{\oplus 8}, \psi_{L}(S)$ is a codimension 8 linear section of $X_{7}$;

- $g=8: X_{8}=\operatorname{Gr}(2,6)$ and $E_{8}=\mathcal{O}_{X_{8}}(1)^{\oplus 6}, \psi_{L}(S)$ is a codimension 6 linear section of $X_{8}$;

- $g=9: X_{9}$ is the Langrangian Grassmannian $\operatorname{LGr}(3,6)$ and $E_{9}=\mathcal{O}_{X_{9}}(1)^{\oplus 4}, \psi_{L}(S)$ is a codimension 4 linear section of $X_{9}$;

- $g=10: X_{10}$ is the flag variety of dimension 5 associated with the adjoint representation of $G_{2}$ embedded in $\mathbb{P}^{13}$ and $E_{10}=\mathcal{O}_{X_{10}}(1)^{\oplus 3} ; \psi_{L}(S)$ is a codimension 3 linear section of $X_{10}$;

- $g=12$ : let $V$ be a 7-dimensional vector space and $X_{12}=\operatorname{Gr}(3, V)$. Then $(S, L) \in$ $\mathcal{K}_{12}$ is BN general if and only if $\psi_{L}(S)$ is birational to a hyperplane section of $\operatorname{Gr}(3, V, N) \subseteq \mathbb{P}^{13}$, where $N \subseteq \wedge^{2} V^{\vee}$ is a nondegenerate 3-dimensional subspace and $\operatorname{Gr}(3, V, N) \subseteq \operatorname{Gr}(3, V)$ consists of 3-dimensional subspaces $U$ of $V$ such that the restriction of $N$ to $U \times U$ is zero.

Here, the nondegeneracy of the subspace $N \subseteq \wedge^{2} V^{\vee}$ means that there is no decomposable vector in $N \wedge V^{\vee} \subseteq \wedge^{3} V^{\vee}$. When $N$ is nondegenerate, $\operatorname{Gr}(3, V, N)$ is a smooth Fano three-fold of index 1 and can be considered as a complete intersection with respect to the vector bundle $\wedge^{2} \mathcal{F}^{\oplus 3}$ on $\operatorname{Gr}(3, V)$, where $\mathcal{F}$ is the dual of the universal subbundle on $\operatorname{Gr}(3, V)$.

Remark 2.6. The homogenous space $X_{g}$ is the quotient of a simply connected, semisimple Lie group by a maximal parabolic subgroup. Here, we list some of the associated semisimple Lie groups of $X_{g}(7 \leq g \leq 10)$, which will be used later:

- $g=7:$ the spin group $\operatorname{Spin}(10)$;

- $g=8$ : the special linear group $P G L(6)$; 
- $g=9:$ the symplectic group $S p(6) ;$

- $g=10$ : the exceptional group of type $G_{2}$.

Next, observing that a smooth codimension 3 linear section of $\operatorname{Gr}(2,5)$ is the unique (up to isomorphism) Fano three-fold $\mathcal{F}_{5}$ of degree 5 and index 2, one can certainly consider the general $\mathrm{K} 3$ surface in $\mathcal{K}_{6}$ as a quadric hypersurface in $\mathcal{F}_{5}$. It is known that the Fano three-fold $\mathcal{F}_{5}$ is a quasi-homogenous space with automorphism group PSL(2). The following lemma describes the locus of such K3 surfaces.

Lemma 2.7. A BN general $\mathrm{K} 3$ surface $(S, L)$ of genus 6 is contained in the smooth Fano three-fold $\mathcal{F}_{5}$ if and only if $(S, L)$ is not contained in $D_{4,0}^{6}$.

Proof. By Theorem 2.5, we already know that a BN general K3 surface $(S, L) \in \mathcal{K}_{6}$ is a complete intersection of a quadric hypersurface $Y \subseteq X_{6}$ and a codimension 3 linear section $\Sigma \subseteq X_{6}$. We know that $\Sigma$ is isomorphic to $\mathcal{F}_{5}$ if $\Sigma$ is smooth.

If $\Sigma$ is singular, we claim that $\Sigma$ must contain a quadric surface $Q$. Actually, the codimension 3 linear sections in $X_{10}$ have been determined by Todd and Kimura (cf. $[13,27])$, and it is straightforward to see the existence of the quadric surface. For instance, a codimension 3 linear section $\Sigma_{0}$ with an $A_{1}$ singularity (this is the generic case) is defined in $\mathbb{P}^{6}$ as follows:

$$
\begin{aligned}
z_{0} z_{2}+z_{1} z_{4}+z_{3}^{2} & =0 \\
z_{0} z_{5}+z_{4}^{2}+z_{3} z_{6} & =0 \\
z_{1} z_{5}-z_{2} z_{4} & =0 \\
z_{1} z_{6}-z_{3} z_{4} & =0 \\
z_{2} z_{6}-z_{3} z_{5} & =0 .
\end{aligned}
$$

Then there exists a quadric cone $Q=\left\{z_{0} z_{2}+z_{3}^{2}=z_{4}=z_{5}=z_{6}=0\right\}$ in $\Sigma_{0}$. The K3 surface $(S, L)$ is contained in $D_{4,0}^{6}$ since the intersection $Q \cap Y$ is an elliptic curve of degree 4 .

\subsection{Non-BN general K3 surfaces}

In this section, we classify all non-BN general K3 surfaces for $6 \leq g \leq 12(g \neq 11)$ and interpret them as a union of NL divisors in $\mathcal{K}_{g}$. This is natural because non-BN general K3 surfaces $(S, L)$ must contain some special curve, and hence lie in some NL divisor $D_{d, n}^{g}$. 
Lemma 2.8. The locus of non-BN general K3 surfaces in $\mathcal{K}_{g}$ is a union of the NL divisors $D_{d, n}^{g}$ satisfying

$$
\sqrt{2(g-1) n}<d \leq \min \left\{g-1, \frac{n+2}{2}+g-\frac{2 g+2}{n+4}\right\} .
$$

In particular, a quasi-polarized K3 surface $(S, L)$ of genus $6 \leq g \leq 12$ and $g \neq 11$ is non-BN general if and only if it lies in one of the following NL divisors:

(I) $D_{d, 0}^{g}, d=1,2, \ldots,\left[\frac{g-1}{2}\right]+1$;

$$
\begin{aligned}
& -g=6, D_{5,2}^{6} \\
& -g=7, D_{5,2}^{7}, D_{6,2}^{7} \\
& -g=8, D_{6,2}^{8}, D_{7,2}^{8} \\
& -g=9, D_{6,2}^{9}, D_{7,2}^{9} \\
& -g=10, D_{7,2}^{10}, D_{8,2}^{10}, D_{9,4}^{10} \\
& -g=12, D_{7,2}^{12}, D_{8,2}^{12}, D_{9,2}^{12}, D_{10,4}^{12}, D_{11,4}^{12}
\end{aligned}
$$

Proof. First, suppose $(S, L)$ is not BN general. Then there exist line bundles $M, N \in$ $\operatorname{Pic}(X)$ satisfying $M+N=L$ and

$$
h^{0}(M) h^{0}(N) \geq g+1
$$

To compute $h^{0}(M)$ and $h^{0}(N)$, let us recall some results about the linear systems on K3 surfaces. Let $F$ be an effective divisor on $S$. Saint-Donat has shown that the following conditions are satisfied:

(i) When $|F|$ has no fixed component, then $|F|$ is basepoint-free and one of the following holds:

- $F^{2}>0, h^{1}(F)=0$. The Riemann-Roch theorem yields

$$
h^{0}(F)=\frac{F^{2}}{2}+2
$$

- $F^{2}=0, F=k E$ for some line bundle $E$ satisfying $E^{2}=0$ and $h^{0}(F)=k+1$.

(ii) When $F=F^{\prime}+\Gamma$ with the fixed component $\Gamma$ and $F^{\prime}$ has no fixed component, then $F^{\prime}$ is either base point free or $F^{\prime}=k E$ by (i). For each connected reduced fixed component $\Gamma^{\prime}$ of $\mathrm{F}$, we have $\left(\Gamma^{\prime}\right)^{2}=-2$ and $F^{\prime} \cdot \Gamma^{\prime}=0$ or 1 if $F^{\prime}$ is base point free; $E \cdot \Gamma^{\prime}=0$ or 1 if $F^{\prime}=k E$. The converse is also true. 
A connected component $\Gamma^{\prime}$ of $\Gamma$ is said to be of Type $\mathrm{I}$ if it is reduced with $\Gamma^{\prime} \cdot F^{\prime}=1$ (respectively, $\Gamma^{\prime} \cdot E=1$ ) and of Type II otherwise. The first cohomology of $F$ vanishes if $F$ only has Type I fixed component and $F^{\prime}$ is base point free.

Coming back to the proof, we discuss two cases.

Case 1. If $|L|$ contains a fixed component, then $S$ is an elliptic K3 surface with a section that lies in $D_{1,0}^{g}$ (see also Section 2.7). The assertion holds.

Case 2. If $|L|$ has no fixed component, we claim that we can find divisors $\tilde{M}, \tilde{N}=L-\tilde{M}$ such that $h^{1}(\tilde{M})=h^{1}(\tilde{N})=0$ and $h^{0}(\tilde{M}) h^{0}(\tilde{N}) \geq h^{0}(L)$. Admitting this, we set $\tilde{M}^{2}=n$ and $L \cdot \tilde{M}=d$; then $h^{0}(\tilde{M})=\frac{n}{2}+2$ and $h^{0}(\tilde{N})=g+\frac{n}{2}+1-d$. Without loss of generality, we assume $h^{0}(\tilde{M}) \leq h^{0}(\tilde{N})$. Thus we obtain

$$
\left\{\begin{array}{l}
\left(\frac{n}{2}+2\right)\left(g+\frac{n}{2}+1-d\right) \geq g+1 \\
g+\frac{n}{2}+1-d \geq \frac{n}{2}+2 \\
d^{2}-n(2 g-2)>0
\end{array}\right.
$$

Here the last inequality in (2.3) is just the Hodge index theorem. Thus $(S, L)$ is contained in $\mathcal{C}_{d, n^{\prime}}^{g}$ where $d, n$ satisfies (2.3).

Now we prove the claim. Note that the inequality $h^{0}(M) h^{0}(L-M) \geq h^{0}(L)$ still holds if we replace $M$ by its base point free part. So we can assume that $M$ is base point free.

Denote by $\Gamma_{\mathrm{I}}\left(\Gamma_{\mathrm{II}}\right)$ the sum of all Type I (Type II) fixed components of $N$. Then we can write $N=N^{\prime}+\Gamma_{\mathrm{I}}+\Gamma_{\mathrm{II}}$ and $N^{\prime}$ has no fixed component. Then the line bundles

$$
\tilde{M}:= \begin{cases}M+\Gamma_{\mathrm{II}} & \text { if } N^{\prime} \text { is base point free } \\ M+(k-1) E+\Gamma_{\mathrm{II}} & \text { if } N^{\prime}=k E \text { and } E^{2}=0,\end{cases}
$$

and $\tilde{N}=L-\tilde{M}$ has zero first cohomology. This is because $L$ is nef, which implies that $\tilde{M}$ and $\tilde{N}$ have no Type II fixed component by a simple intersection computation. Thus we have proved the claim.

Moreover, our assertion follows easily from the fact that the union of $\mathcal{C}_{d, n}^{g}$ is the same as the union of $D_{d, n}^{g}$ for $d, n$ satisfying the condition (2.3). 
Conversely, if $(S, L)$ lies in one of the NL divisors listed in the statement, then $\operatorname{Pic}(S)$ contains an element $M$ such that $M \cdot L=d$ and $M^{2}=n$, satisfying the given condition. We have the inequality

$$
h^{0}(M) h^{0}(L-M) \geq\left(\frac{M^{2}}{2}+2\right)\left(\frac{(L-M)^{2}}{2}+2\right) \geq g+1 .
$$

Remark 2.9. A similar computation can be found in [12, Chapter 5], where Johnsen and Knutsen classify all non-Clifford general K3 surfaces of genus $6 \leq g \leq 10$.

\subsection{Projective model of non-BN general K3 surfaces}

In this section, we describe the projective models of non-BN general K3 surfaces, which will be used later. Indeed, there is a projective model for the general K3 surface in each of the NL divisors of Lemma 2.6. Let us start with Saint-Donat's result.

Proposition 2.10 ([24]). Assume that $L$ is ample. If $\psi_{L}$ is not isomorphic to its image, then

(1) $|L|$ has a fixed component $D$, which is a smooth rational curve. The image of $\psi_{|L-D|}(S)$ is a rational normal curve of degree $g$ in $\mathbb{P}^{g} ;$

(2) $\psi_{L}$ is a generically 2:1 map and $\psi_{L}(S)$ is a smooth rational normal scroll of degree $g-1$, or a cone over a rational normal curve of degree $g+1$.

The first (respectively, second) case happens if and only if ( $S, L)$ admits an elliptic fibration with a section (respectively, bisection), which means that $S$ contains an elliptic curve of degree 1 (respectively, 2). Moreover, we have the following lemma.

Lemma 2.11. For $2 \leq g \leq 12$ and $g \neq 11$, the polarized $K 3$ surface $(S, L)$ lies in $\mathcal{C}_{2,0}^{g}$ if and only if $\psi_{L}: S \rightarrow \mathbb{P}^{g}$ is not isomorphic to its image. Moreover, $\mathcal{C}_{2,0}^{g}=D_{1,0}^{g} \cup D_{2,0}^{g}$ when $g \neq 7$, and $\mathcal{C}_{2,0}^{7}=D_{1,0}^{g} \cup D_{2,0}^{g} \cup D_{5,2}^{7}$.

Proof. By the discussion above, $\psi_{L}$ is not an isomorphism if and only if it contains an elliptic curve of degree 1 or 2 . The locus of those $(S, L) \in \mathcal{K}_{g}$ containing an elliptic curve of degree 1 is the irreducible divisor $D_{1,1}^{g}$ because the corresponding rank 2 sublattice

$$
\left(\begin{array}{ll}
g & 1 \\
1 & 0
\end{array}\right)
$$

is always primitive. 
Similarly, for $6 \leq g \leq 12$ and $g \neq 11$, the locus of those $(S, L) \in \mathcal{K}_{g}$ containing an elliptic curve of degree 2 is the irreducible divisor $D_{2,1}^{g}$, except in the case $g=12$. When $g=12$, this locus is the union of the two irreducible divisors $D_{2,1}^{7}$ and $D_{5,2}^{7}$, because there are two primitive lattices

$$
\left(\begin{array}{cc}
12 & 2 \\
2 & 0
\end{array}\right) \text { and }\left(\begin{array}{cc}
12 & 5 \\
5 & 2
\end{array}\right)
$$

which contain an element $\beta$ satisfying $\beta^{2}=0$ and $\beta \cdot L=2$.

For the case $(S, L)$ is non-BN general and $\psi_{L}$ is an isomorphism, the image $\psi_{L}(S)$ is contained in some rational normal scrolls $\mathcal{T}$, that is, projective bundles over $\mathbb{P}^{1}$. Here we say a rational number scroll $\mathcal{T}$ is of type $\left(d_{1}, d_{2}, \ldots d_{n}\right)$ if

$$
\mathcal{T} \cong \mathbb{P}\left(\bigoplus_{i=1}^{n} \mathcal{O}_{\mathbb{P}^{1}}\left(d_{i}\right)\right)
$$

for $d_{i} \geq 0$. The image of the natural morphism $\mathcal{T} \rightarrow \mathbb{P}\left(H^{0}\left(\bigoplus_{i=1}^{n} \mathcal{O}_{\mathbb{P}^{1}}\left(d_{i}\right)\right)\right)$ may be singular if $d_{i}=0$ for some $i$, and we say that $\mathcal{T}$ is a singular rational normal scroll.

For the low genera appearing in this paper, Johnsen and Knutsen [12] and Hana [8] have classified all the non-BN general K3 surfaces whose projective models lie in various rational normal scrolls. Using their results, one can find the projective models of the K3 surfaces lying in each irreducible component of the non-BN general locus. For instance, when $g=6$, we have

- $(S, L) \in D_{3,0}^{6}$ if $\psi_{L}(S)$ is the hypersurface of a rational normal scroll of type $(2,1,1)$;

- $(S, L) \in D_{5,2}^{6}$ if $\psi_{L}(S)$ is the complete intersection of a singular rational normal scroll of type $(2,1,0,0)$.

We refer the readers to [12, Chapter $11 ; 8$, Chapter 2] for the complete list of all possible projective models.

\section{Birational Models of $\mathcal{K}_{g}$}

This section is devoted to the description of a birational model of $\mathcal{K}_{g}$ via GIT. More precisely, we can interpret the moduli space of BN general K3 surfaces as a moduli space of smooth complete intersections, using Theorem 2.5. The latter can be constructed via GIT and this allows us to compute the Picard group of $\mathcal{K}_{g}$ directly. 


\subsection{GIT construction}

Recall that the general $\mathrm{K} 3$ surface in $\mathcal{K}_{g}$ among the range of Mukai models is a complete intersection with respect to some vector bundle $E_{g}$ on a homogenous space $X_{g}$, by Theorem 2.5. We obtain the GIT model of the moduli space as follows:

(I) For $6 \leq g \leq 10$, let $V_{g}=H^{0}\left(X_{g}, \mathcal{O}_{g}(1)\right)$, the complete intersections $\psi_{L}(S)$ are parameterized by Grassmannians with a natural group action of $G_{g}$ coming from the action on $X_{g}$ :

- $W_{6}=\mathbb{P}\left(H^{0}\left(F_{5}, \mathcal{O}_{\mathcal{F}_{5}}(2)\right)\right), G_{6}=P S L(2) ;$

- $W_{7}=\mathrm{Gr}\left(8, V_{7}\right), G_{7}=\operatorname{Spin}(10)$;

- $W_{8}=\mathrm{Gr}\left(6, V_{8}\right), G_{8}=S L(6)$;

- $W_{9}=\mathrm{Gr}\left(4, V_{9}\right), G_{9}=S p(6)$;

- $W_{10}=\operatorname{Gr}\left(3, V_{10}\right), G_{10}$ is the quotient of $G_{2}$ by its center;

As $W_{g}$ has Picard number 1 , one can naturally take the GIT quotient $W_{g}^{s s} / / G_{g}$ with respect to the $G_{g}$-linearized ample line bundle $\mathcal{O}_{W_{g}}(1)$, where $W_{g}^{s s}$ is the semistable locus of $W_{g}$. We denote by

$$
\Phi_{g}: W_{g}^{s s} / / G_{g}-\rightarrow \mathcal{K}_{g}
$$

the natural rational map to $\mathcal{K}_{g}$. As proved by Mukai (cf. [17, Theorem 0.2]), $\Phi_{g}$ is birational, and thus the image of $\Phi_{g}$ contains an open subset of $\mathcal{K}_{g}$. Our goal of this section is to describe the image of smooth complete intersections in $\mathcal{K}_{g}$ via $\Phi_{g}$.

(II) The case of $g=12$ is slightly different. The BN general K3 surface is a hyperplane section of the smooth Fano three-fold $\operatorname{Gr}(3, V, N) \subseteq \mathbb{P}^{13}$ for some nondegenerate $N$. This is a complete intersection with respect to the vector bundle $\left(\wedge^{2} \mathcal{F}\right)^{\oplus 3}$ on $X_{12}=\operatorname{Gr}(3, V)$. Since $H^{0}\left(\operatorname{Gr}(3, V), \wedge^{2} \mathcal{F}\right) \cong \wedge^{2} V^{\vee}$, we have a natural parameter space $\mathcal{V}_{12}$ of nondegenerate $\operatorname{Gr}(3, V, N)$ which is birational to the GIT quotient

$$
\operatorname{Gr}\left(3, \wedge^{2} V^{\vee}\right)^{s s} / / P G L(V) .
$$

The moduli space $\mathcal{K}_{12}$ is birational to the $\mathbb{P}^{13}$-bundle $\mathcal{P}_{12} \rightarrow \mathcal{V}_{12}$, where the fiber over an element $F \in \mathcal{V}_{12}$ is the projective space $\mathbb{P}\left(H^{0}\left(\mathcal{O}_{F}(1)\right)\right)$. In the rest of this section, we will discuss the GIT stability of the smooth elements in $W_{g}$. We start with the study of discriminant loci of complete intersections, which play an important role in the proof of the main theorem. 


\subsection{Discriminant loci of complete intersections}

First, we discuss general discriminant loci in moduli of complete intersections with respect to vector bundles, and then we restrict to our cases.

Let $X$ be a smooth projective variety and $E$ be a globally generated vector bundle on $X$. The discriminant locus $\Delta_{E}$ of $E$ is defined by

$$
\Delta_{E}:=\left\{s \in \mathbb{P}\left(H^{0}(X, E)\right)^{\vee} \mid(s)_{0} \text { is either singular, or not a complete intersection w.r.t. } E\right\},
$$

when $E$ is a very ample line bundle, $\Delta_{E}$ is isomorphic to the dual variety $X^{\vee}$, which is an irreducible subvariety in $\mathbb{P}\left(H^{0}(X, E)\right)$. Moreover, $\Delta_{E}$ is an irreducible divisor if there exists an element $s \in \Delta_{E}$ such that $(s)_{0}$ has only isolated singularities (cf. [6, Proposition 1.3]). This implies the following result for vector bundles.

Proposition 3.1. Let $\mathbb{P}(E)$ be the associated projective bundle on $X$, and $\mathcal{O}_{\mathbb{P}(E)}(1)$ be its relatively ample line bundle. There is a natural isomorphism

$$
\Psi: H^{0}\left(\mathbb{P}(E), \mathcal{O}_{\mathbb{P}(E)}(1)\right) \stackrel{\sim}{\rightarrow} H^{0}(X, E) .
$$

The zero locus of a nonzero section $s \in H^{0}(\mathbb{P}(E), \mathcal{O}(1))$ in $\mathbb{P}(E)$ is smooth if and only if the section $\Psi(s) \in H^{0}(X, E)$ is nondegenerate and the zero locus of $\Psi(s)$ is smooth in $\mathbb{P}(E)$. In other words, $\Delta_{E}$ is isomorphic to $\Delta_{\mathcal{O}_{\mathbb{P}(E)}(1)}$. If $E$ is very ample, that is, $\mathcal{O}_{\mathbb{P}(E)}(1)$ very ample, then $\Delta_{E}$ is an irreducible divisor when there is a complete intersection with respect to $E$ with only isolated singularities.

Proof. For the proof, see [18, Proposition 1.9].

In the case where $E=L^{\oplus k}$ is a direct sum of $k$-copies of a very ample line bundle $L$, one can also parameterize the complete intersections with respect to $E$ by the Grassmannian $\operatorname{Gr}\left(k, H^{0}(X, L)\right)$ and define

$$
\begin{aligned}
\Delta_{k, L}=\left\{\left\langle s_{1}, \ldots, s_{k}\right\rangle \in \operatorname{Gr}\left(k, H^{0}(X, L)\right) \mid\left(\oplus_{i} s_{i}\right)_{0}\right. \text { is either singular or not a } \\
\text { complete intersection }\}
\end{aligned}
$$

which is called the discriminant locus of $L^{\oplus k}$ in $\operatorname{Gr}\left(k, H^{0}(X, L)\right)$. 
Proposition 3.2. The discriminant locus $\Delta_{k, L}$ is an irreducible divisor in $\operatorname{Gr}\left(k, H^{0}(X, L)\right)$ when there is a complete intersection with respect to $E$ with at worst isolated singularities.

Proof. The assertion follows from the fact that $\Delta_{k, L}$ is the quotient $\left(\Delta_{E} \cap\right.$ $\left.\mathbb{P}\left(H^{0}(X, E)\right)^{S}\right) / S L(k)$.

Remark 3.3. In this paper, one can see that most of the vector bundles $E_{g}$ in Mukai's description are very ample, so the irreducibility of the discriminant locus follows from Proposition 3.1. We should also mention that this method can still be applied to study the case of K3 surfaces of genus 18 and 20 (cf. [18]), although the corresponding vector bundles are not ample.

\subsection{GIT stability}

Now we discuss GIT stability of the smooth complete intersections in Section 3.1. With the notation in Theorem 2.5, we say that a nondegenerate three-fold $\operatorname{Gr}(3, V, N)$ is nonspecial if it does not belong to Prokhorov's class of genus 12 Fano three-fold defined in [23], and is special otherwise. Then we start with a useful lemma.

Lemma 3.4. Let $X$ be either a K3 surface or a nonspecial smooth nondegenerate threefold $\operatorname{Gr}(3, V, N)$. Let $L$ be the natural polarization on $X$. The group $\operatorname{Aut}_{L}(X)$ of automorphisms $f: X \stackrel{\sim}{\rightarrow} X$ that preserve the polarization $L$, that is, $f^{*} L \cong L$, is finite.

Proof. For the proof, see [11, Chapter 5, Proposition 3.3] for K3 surfaces and [23] for automorphism groups of Fano three-folds.

Remark 3.5. By [23], there are only three types of Fano three-folds of genus 12 with infinite automorphism group. One is the Mukai-Umemura manifold constructed in [20] with automorphism group $S L(2)$, and the other two have automorphism groups $G_{a}$ and $G_{m}$. The moduli space for each of these types is at most 1-dimensional.

Theorem 3.6. For $6 \leq g \leq 10$, if $S \in W_{g}$ is one of the smooth complete intersections described above, then $S$ is GIT stable in $W_{g}$. Moreover, the smooth nondegenerate complete intersection $\operatorname{Gr}(3, V, N) \subseteq \operatorname{Gr}(3, V)$ is GIT stable in $\operatorname{Gr}\left(3, \wedge^{2} V^{\vee}\right)$ if it is not in the orbit of special complete intersections. 
Proof. Let $\Delta_{g} \subseteq W_{g}$ denote the discriminant locus, in the sense of (3.3), for $6 \leq g \leq 10$. By Lemma 3.2, $\Delta_{g}$ is an irreducible hypersurface in $W_{g}$, since there are complete intersections with only one rational double point. Indeed, the K3 surface with a primitive Picard lattice

$$
\left(\begin{array}{cc}
2 g-2 & 0 \\
0 & -2
\end{array}\right)
$$

will be such a singular complete intersection.

The discriminant locus $\Delta_{g}$ is thus cut out by a single homogenous equation $\Omega_{g}=$ 0 , namely the discriminant form. Moreover, the discriminant form $\Omega_{g}$ is $G_{g}$-invariant because the property of singularity is preserved under changes of coordinates. Hence $\Omega_{g}$ is a $G_{g}$-invariant function. The semistability of the smooth surface $S$ then follows from the fact that $\Omega_{g}$ does not vanish at $S$.

By Lemma 3.4, we know that $S$ only have finite stabilizers. Suppose that $S$ is strictly semistable and hence the orbit of $S$ is not closed in $W_{g}-\Delta_{g}$. We claim that there must exist an element $S^{\prime} \in W_{g}-\Delta_{g}$ in the closure of the orbit of $S$ such that $S^{\prime}$ has stabilizer group of strictly positive dimension. Note that $W_{g}-\Delta_{g}$ is affine with the natural $G_{g}$ action, one can just take $S^{\prime}$ to be a point in the closure of the orbit of $S$ in $W_{g}-\Delta_{g}$ and not contained in the orbit itself, which must then have a strictly positive-dimensional stabilizer group. The surface $S^{\prime}$ is a smooth K3 surface with an infinite automorphism group preserving the polarization, which contradicts Lemma 3.4. Therefore, $S$ must be GIT stable.

For the case $g=12$, we employ a similar idea, using a discriminant form. Let $W_{12} \subseteq \operatorname{Gr}\left(3, \wedge^{2} V^{\vee}\right)$ be the locus of nondegenerate elements in $\operatorname{Gr}\left(3, \wedge^{2} V\right)$ and $\Delta:=$ $\operatorname{Gr}\left(3, \wedge^{2} V^{\vee}\right) \backslash W_{12}$ be the complement of $W_{12}$. As any smooth nondegenerate $\operatorname{Gr}(3, V, N)$ has only finitely many automorphisms if it is nonspecial, it suffices to show that $\Delta$ is an irreducible $G_{12}$-invariant divisor in $\operatorname{Gr}\left(3, \wedge^{2} V^{\vee}\right)$. To prove this, let us consider the incident variety

$$
\Omega=\{(N,[v]) \mid \exists \omega \in N \text { s.t. } \omega \wedge v \text { is decomposable }\} \subseteq \mathrm{Gr}\left(3, \wedge^{2} V^{\vee}\right) \times \mathbb{P}\left(V^{\vee}\right),
$$

with the first projection $\pi_{1}(\Omega)=\Delta$. The second projection $\pi_{2}: \Omega \rightarrow \mathbb{P}\left(V^{\vee}\right)$ is surjective, and the fiber $\pi_{2}^{-1}(v)$ for any $v \in \mathbb{P}\left(V^{\vee}\right)$ can be described as follows.

Set $D_{v}=\left\{[\omega] \in \mathbb{P}\left(\wedge^{2} V^{\vee}\right) \mid \omega \wedge v\right.$ is decomposable $\} \subseteq \mathbb{P}\left(\wedge^{2} V^{\vee}\right)$, and $V_{v}=V^{\vee} / v$ the quotient vector space. Then $D_{v}$ is isomorphic to the 14-dimensional irreducible variety

$$
\left(\widetilde{\mathrm{Gr}}\left(2, V_{v}\right) \times V_{v}\right) / \mathbb{C}^{*},
$$


where $\widetilde{G r}\left(2, V_{v}\right)$ is the space of decomposable vectors in $\wedge^{2} V_{v}$, and $\mathbb{C}^{*}$ acts simultaneously by scaling on $\widetilde{\mathrm{Gr}}\left(2, V_{v}\right)$ and $V_{v} \cong v \wedge V_{v}$.

Next, we define the correspondence

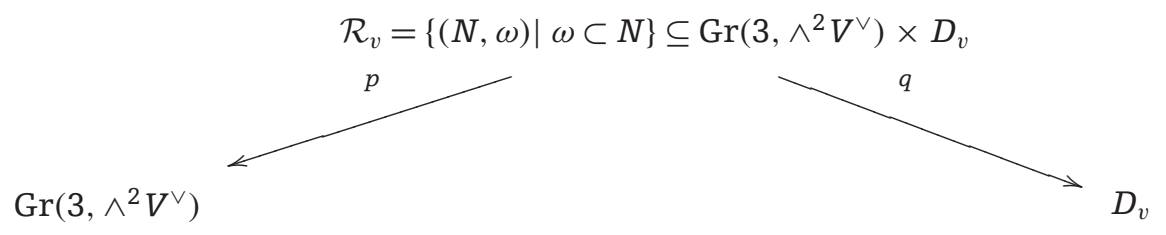

where the fiber of the second projection $q$ over $\omega \in D_{v}$ is isomorphic to the Grassmannian $\operatorname{Gr}\left(2, \wedge^{2} V^{\vee} / \omega\right)$. Now we claim that the first projection $p$ is generically finite, and then we have $\pi_{2}^{-1}(v)=p\left(\mathcal{R}_{v}\right)$ is irreducible of dimension 50 .

To prove the claim, we need only show the existence of a fiber of $p$ which is finite. Actually, it is easy to see that there exists a 3-dimensional subspace $N \subseteq \wedge^{2} V_{v}^{\vee}$ such that the plane $\mathbb{P}(N)$ meets $\operatorname{Gr}\left(2, V_{v}^{\vee}\right)$ in $\mathbb{P}\left(\wedge^{2} V_{v}^{\vee}\right)$ at finitely many points. Identifying $N$ as an element in $\operatorname{Gr}\left(3, \wedge^{2} V^{\vee}\right)$, we know that the fiber $p^{-1}(N)$ is just the intersection of $\mathbb{P}(N)$ and $\operatorname{Gr}\left(2, V_{v}^{\vee}\right)$ in $\mathbb{P}\left(\wedge^{2} V_{v}^{\vee}\right)$, which is finite. Thus, we have proved the claim, and we have that $\Omega$ is irreducible of dimension 56 .

For the rest of the assertion, we still need to understand the general fiber of the projection $\pi_{1}: \Omega \rightarrow \operatorname{Gr}\left(3, \wedge^{2} V^{\vee}\right)$. Indeed, for general $N \in \Delta$, it contains only one 1dimensional linear subspace spanned by an element $\omega$ of the form

$$
\omega=f_{1} \wedge f_{2}+v \wedge f_{3}
$$

for some $v \in V^{\vee}$, and $f_{1}, f_{2}, f_{3} \in V_{v}$ are linearly independent. Then the fiber $\pi_{1}^{-1}(N)$ is just the 3-dimensional projective space $\mathbb{P}\left(\left\langle v, f_{1}, f_{2}, f_{3}\right\rangle\right)$.

Combining everything, we get $\Delta=\pi_{1}(\Omega)$ is irreducible of dimension 53 in $\mathrm{Gr}\left(3, \wedge^{2} V^{\vee}\right)$, and this completes the proof.

\section{Proof of the Main Theorem}

According to Borcherds' theta lifting theory, a beautiful result of the NL theory on K3 surfaces is that the generating series of NL divisors (in the sense of [16]) is a vectorvalued modular form (cf. [2, 16]). As an application, Bruinier [4] computes the dimension of the subspace $\operatorname{Pic}_{\mathbb{Q}}^{N L}\left(\mathcal{K}_{g}\right)$ of $\operatorname{Pic}_{\mathbb{Q}}\left(\mathcal{K}_{g}\right)$ generated by NL divisors (see also [15]). 
Lemma 4.1 ([15]). Let $\rho_{g}$ be the dimension of $\operatorname{Pic}_{\mathbb{Q}}^{N L}\left(\mathcal{K}_{g}\right)$. Then

$$
\rho_{g}=\frac{31 g+24}{24}-\frac{1}{4} \alpha_{g}-\frac{1}{6} \beta_{g}-\sum_{k=0}^{g-1}\left\{\frac{k^{2}}{4 g-4}\right\}-\sharp\left\{k \mid \frac{k^{2}}{4 g-4} \in \mathbb{Z}, 0 \leq k \leq g-1\right\}
$$

where

$$
\alpha_{g}=\left\{\begin{array}{ll}
0, & \text { if } g \text { is even; } \\
\left(\frac{2 g-2}{2 \ell-3}\right) & \text { otherwise. }
\end{array}, \quad \beta_{g}= \begin{cases}\left(\frac{g-1}{4 g-5}\right)-1, & \text { if } g \equiv 1 \bmod 3, \\
\left(\frac{g-1}{4 g-5}\right)+\left(\frac{g-1}{3}\right) & \text { otherwise }\end{cases}\right.
$$

and $\left(\frac{a}{b}\right)$ is the Jacobi symbol. In particular, $\rho_{g}=6,7,7,8,9,12$ for $g=6,7,8,9,10,11$.

Now we are ready to give the proof of our main theorem.

Proof. We still separate the proof into two cases: (I) $6 \leq g \leq 10$ and (II) $g=12$. For case (I), we let $\mathcal{U}_{g} \subseteq W_{g}$ be the open subset parameterizing the smooth complete intersections. By definition, $\mathcal{U}_{g}$ is the complement of the discriminant locus $\Delta_{g}$ in $W_{g}$. By Theorem 3.6, the reductive Lie group $G_{g}$ acts properly on $\mathcal{U}_{g}$. Then we get an open immersion:

$$
\Phi_{g}^{\prime}: \mathcal{U}_{g} / / G_{g} \longrightarrow \mathcal{K}_{g}
$$

We denote by $\mathcal{K}_{g}^{B N}$ the complement of the union of NL divisors listed in Theorem 1.1. Then it is contained in the image of $\Phi_{g}^{\prime}$ by Theorem 2.5 and Lemma 2.8.

Moreover, since the complement $W_{g} \backslash \mathcal{U}_{g}$ is an irreducible divisor and $\operatorname{Pic}\left(W_{g}\right) \cong$ $\mathbb{Z}$, the Picard group of $\mathcal{U}_{g}$ is torsion. Let $\operatorname{Pic}_{\mathbb{Q}}\left(\mathcal{U}_{g}\right)_{G_{g}}$ be the group of $G_{g}$-linearized line bundles on $\mathcal{U}_{g}$. By [14, Proposition 4.2], there is an injection

$$
\operatorname{Pic}\left(\mathcal{U}_{g} / / G_{g}\right) \hookrightarrow \operatorname{Pic}\left(\mathcal{U}_{g}\right)_{G_{g}} .
$$

The forgetful map $\operatorname{Pic}_{\mathbb{Q}}\left(\mathcal{U}_{g}\right)_{G_{g}} \rightarrow \operatorname{Pic}_{\mathbb{Q}}\left(\mathcal{U}_{g}\right)$ has kernel the group of rational characters $\chi\left(G_{g}\right)$, which is trivial because $G_{g}$ is simple. Thus $\operatorname{Pic}_{\mathbb{Q}}\left(\mathcal{U}_{g} / / G_{g}\right)$ is trivial. Note that $\mathcal{K}_{g}^{B N}$ is an open subset of $\mathcal{U}_{g} / / G_{g}$; then $\operatorname{Pic}_{\mathbb{Q}}\left(\mathcal{U}_{g} / / G_{g}\right)=0$ implies that Pic $\mathbb{Q}_{\mathbb{Q}}\left(\mathcal{K}_{g}^{B N}\right)=0$ by the localization sequence for Picard groups. It follows that the Picard group $\operatorname{Pic}_{\mathbb{Q}}\left(\mathcal{K}_{g}\right)$ is spanned by precisely the NL divisors stated in the theorem. Finally, these NL divisors form a basis of $\operatorname{Pic}_{\mathbb{Q}}\left(\mathcal{K}_{g}\right)$ by the dimension counts of Lemma 4.1.

For case (II), we let $\mathcal{U}_{12} \subseteq \mathcal{P}_{12}$ be the locus parameterizing complete intersections of a smooth three-fold $\operatorname{Gr}(3, V, N) \subseteq \mathbb{P}^{13}$ which are not in the orbit of special three-folds 
and a hyperplane section in $\mathbb{P}^{13}$. By Theorem 3.6, we know that $\mathcal{U}_{12}$ admits a fibration to the orbit space

$$
W_{12}^{n s} / / S L(V)
$$

where $W_{12}^{n s} \subseteq \operatorname{Gr}\left(3, \wedge^{2} V^{\vee}\right)$ is the parameter space of $\operatorname{Gr}(3, V, N)$ which is not in the orbit of special three-folds. The open subset

$$
W_{12}^{n s} \hookrightarrow \operatorname{Gr}\left(3, \wedge^{2} V^{\vee}\right) \backslash \Delta
$$

has Picard number zero, and so does the quotient $W_{12}^{n s} / S L(V)$. The fiber of $\mathcal{U}_{12} \rightarrow$ $W_{12}^{n s} / S L(V)$ over a three-fold $F \in W_{12}^{n s} / S L(V)$ is the complement of the discriminant $\Delta_{\mathcal{O}_{F}(1)}$ in $\mathbb{P}\left(\mathcal{O}_{F}(1)\right)$. Therefore, $\operatorname{dim}_{\operatorname{Pic}_{\mathbb{Q}}}\left(\mathcal{U}_{12}\right)=0$.

Similarly, one observes that the image of $\mathcal{U}_{12} \hookrightarrow \mathcal{K}_{12}$ contains the complement of the NL divisors listed in the main theorem statement, union with a high codimension $(\geq 2)$ subvariety (see Remark 3.5) parameterizing the K3 surfaces in special three-folds. As before, this shows that the Picard group $\operatorname{Pic}_{\mathbb{Q}}\left(\mathcal{K}_{12}\right)$ is spanned by the NL divisors in the assertion.

By Lemma 4.1, the rank of $\operatorname{Pic}_{\mathbb{Q}}\left(\mathcal{K}_{12}\right)$ is 11 , hence the generators in (1.2) are not linearly independent. They will satisfy a linear relation (4.4) (see Remark 4.2).

Remark 4.2. As we have mentioned at the beginning of this section, the NL divisors $D_{d, n}^{g}$ (in the sense of [16]) correspond to certain vector-valued modular forms (cf. [3, Section 1.2]). So in principle, one can compute the relations between NL divisors via these vector-valued modular forms. In a forthcoming result of Arie Peterson, he shows the following relation on $\operatorname{Pic}_{\mathbb{Q}}\left(\mathcal{K}_{12}\right)$ :

$$
3 D_{8,2}^{12}-D_{9,2}^{12}-4 D_{10,4}^{12}+2 D_{11,4}^{12}+8 D_{4,0}^{12}-5 D_{5,0}^{12}+D_{6,0}^{12}=0 .
$$

Remark 4.3. As shown in [5, Section 6.3] (see also [15] for the more general case), the second cohomology group $H^{2}\left(\mathcal{D}_{g} / \Gamma_{g}, \mathbb{Q}\right)$ has a pure Hodge structure and we have an isomorphism

$$
\operatorname{Pic}_{\mathbb{Q}}\left(\mathcal{D}_{g} / \Gamma_{g}\right) \stackrel{\sim}{\rightarrow} H^{2}\left(\mathcal{D}_{g} / \Gamma_{g}, \mathbb{Q}\right)
$$

from the exponential exact sequence. The latter is also isomorphic to the arithmetic group cohomology $H^{2}\left(\Gamma_{g}, \mathbb{Q}\right)$. The NL conjecture is equivalent to the cohomology group of $\mathcal{D}_{g} / \Gamma_{g}$ being spanned by (classes of) locally Hermitian symmetric subvarieties. Recently, 
there have emerged more standard ways to study this problem on locally Hermitian symmetric varieties of orthogonal type; cf. [1, 10]. In a sequel to this paper, we will approach the conjecture from this direction.

\section{Acknowledgements}

We have benefited from conversations with Brendan Hassett and Jun Li. We are very grateful to Arie Peterson for his useful comments and providing us the relation of NL divisors in Remark 4.2. We would also like to thank the referees for all their comments and suggestions.

\section{References}

[1] Bergeron, N., J. Millson, and C. Moeglin. "Hodge type theorems for arithmetic manifolds associated to orthogonal groups." (2011): preprint. arXiv:1110.3049.

[2] Borcherds, R. E. "The Gross-Kohnen-Zagier theorem in higher dimensions." Duke Mathematical Journal 97, no. 2 (1999): 219-33.

[3] Bruinier, J. H. Borcherds Products on $\mathrm{O}(2, l)$ and Chern Classes of Heegner Divisors, Lecture Notes in Mathematics 1780. Berlin: Springer, 2002.

[4] Bruinier, J. H. "On the rank of Picard groups of modular varieties attached to orthogonal groups." Compositio Mathematica 133, no. 1 (2002): 49-63.

[5] Clozel, L. and T. N. Venkataramana. "Restriction of the holomorphic cohomology of a Shimura variety to a smaller Shimura variety." Duke Mathematical Journal 95, no. 1 (1998): 51-106.

[6] Gelfand, I. M., M. M. Kapranov, and A. V. Zelevinsky. Discriminants, Resultants, and Multidimensional Determinants. Mathematics: Theory \& Applications. Boston, MA: Birkhäuser, Inc., 1994.

[7] Gritsenko, V., K. Hulek, and G. K. Sankaran. "Abelianisation of orthogonal groups and the fundamental group of modular varieties." Journal of Algebra 322, no. 2 (2009): 463-78.

[8] Hana, G. M. "Projective models of Algebraic surfaces in scrolls." PhD thesis, University of Bergen, Norway, 2002.

[9] Harer, J. "The second homology group of the mapping class group of an orientable surface." Inventiones Mathematicae 72, no. 2 (1983): 221-39.

[10] He, H. and J. W. Hoffman. "Picard groups of Siegel modular 3-folds and $\theta$-liftings." Journal of Lie Theory 22, no. 3 (2012): 769-801.

[11] Huybrechts, D. "Lectures on K3 surfaces." www.math.uni-bonn.de/people/huybrech/ K3Global.pdf.

[12] Johnsen, T. and A. L. Knutsen. " $K 3$ Projective Models in Scrolls." Lecture Notes in Mathematics 1842. Berlin: Springer, 2004. 
[13] Kimura, T. "The $b$-functions and holonomy diagrams of irreducible regular prehomogeneous vector spaces." Nagoya Mathematical Journal 85 (1982): 1-80.

[14] Knop, F., H. Kraft, and T. Vust. "The Picard Group of a G-variety." Algebraische Transformationsgruppen und Invariantentheorie, 77-87. DMV Sem. 13. Basel: Birkhäuser, 1989.

[15] Li, Z. and Z. Tian. "Picard groups of moduli spaces of low degree K3 surfaces." (2013): preprint. arXiv:1304.3219.

[16] Maulik, D. and R. Pandharipande. "Gromov-Witten Theory and Noether-Lefschetz Theory." A Celebration of Algebraic Geometry, 469-507. Clay Math. Proc. 18. Providence, RI: American Mathematical Society, 2013.

[17] Mukai, S. "Curves, K3 Surfaces and Fano 3-Folds of Genus $\leq 10$." Algebraic Geometry and Commutative Algebra, vol. I, 357-77. Tokyo: Kinokuniya, 1988.

[18] Mukai, S. "Polarized K3 Surfaces of Genus 18 and 20." Complex Projective Geometry (Trieste, 1989/Bergen, 1989), 264-76. London Mathematical Society Lecture Note Series 179. Cambridge: Cambridge University Press, 1992.

[19] Mukai, S. "New developments in the theory of Fano threefolds: vector bundle method and moduli problems [translation of Sūgaku 47 (1995), no. 2, 125-44; MR1364825 (96m:14059)]." Sugaku Expositions 15, no. 2 (2002): 125-50. Sugaku expositions.

[20] Mukai, S. and H. Umemura. "Minimal Rational Threefolds." Algebraic Geometry (Tokyo/Kyoto, 1982), 490-518. Lecture Notes in Mathematics 1016. Berlin: Springer, 1983.

[21] O'Grady, K. G. "On the Picard group of the moduli space for $K$-3 surfaces." Duke Mathematical Journal 53, no. 1 (1986): 117-24.

[22] O'Grady, K. G. "Moduli of abelian and K3 surfaces." ProQuest LLC, Ann Arbor, MI. PhD thesis, Brown University, 1986.

[23] Prokhorov, Yu. G. "Automorphism groups of Fano 3-folds." Uspekhi Matematicheskikh Nauk 45, no. 3(273) (1990): 195-6.

[24] Saint-Donat, B. "Projective models of $K-3$ surfaces." American Journal of Mathematics 96 (1974): 602-39.

[25] Shah, J. "A complete moduli space for $K 3$ surfaces of degree 2." Annals of Mathematics (2) 112, no. 3 (1980): 485-510.

[26] Shah, J. "Degenerations of $K 3$ surfaces of degree 4." Transactions of the American Mathematical Society 263, no. 2 (1981): 271-308.

[27] Todd, J. A. "The locus representing the lines of four-dimensional space and its application to linear complexes in four dimensions." Proceedings of the London Mathematical Society S2-30, no. 1: 513 . 